\title{
Strength Pareto Evolutionary Algorithm for the Dynamic Economic Emission Dispatch Problem incorporating Wind Farms and Energy Storage Systems
}

\author{
Khalid Alqunun \\ College of Engineering \\ University of Hail \\ Hail, Saudi Arabia \\ khalid.alqunun@gmail.com
}

\begin{abstract}
Intermittent and stochastic characteristics of wind energy sources cause many challenges for the existing power networks. One of these challenges is the violation of the energy balance constraint due to the high penetration of wind power. The use of Energy Storage Systems (ESSs) can facilitate the high penetration of wind power and mitigate the effect of its intermittency. Within this context, ESS incorporate the Dynamic Economic Emission Dispatch (DEED) problem. The problem is formulated as a multi-objective problem and the Strength Pareto Evolutionary Algorithm (SPEA) is used for its resolution. Simulations were carried out on a well-known ten-unit system and the results show the importance of using ESSs in reducing the total production cost of electricity and total emissions.
\end{abstract}

Keywords-dynamic dispatch; wind energy; energy storage systems; evolutionary algorithm

\section{INTRODUCTION}

Due to the energy shortage and the increasing pressure to protect the environment, wind energy is gaining attention. Wind is considered a clean energy source and can mitigate the dependence on fossil fuels. However, the random characteristics of the wind speed result in volatility and uncertainty of the wind power output. Consequently, high penetration of wind power may cause a negative impact on the system stability and lead to the violation of the energy balance constraint [1]. In fact, once the wind power penetration becomes more than $5 \%$ of the total energy production, the power quality will be influenced by the uncertainty of the wind power [2]. Therefore, in wind-thermal systems, it is important to allocate perfectly the generation of all units including wind power in order to alleviate wind power curtailment. This problem is referred to as the power dispatch problem. Several research works have handled the optimal dispatch for the windthermal systems. The resolutions of such problems have been based on quadratic programming, Genetic Algorithm (GA) [3], Particle Swarm Optimization (PSO) [4], simulated annealing [5], harmony search [6], firefly algorithm [7], chemical reaction optimization [8], etc. The uncertainty of the wind power output is handled with different manners, such as scenario method [9], forecast error method [10], stochastic programming [11], probability theory-based model [12], fuzzy logic [13], and chance constraint model [14]. For example, a chance constraint-based formulation for the dispatch problem was described in [15]. In [16], the intermittent nature of the wind power was described by evaluating its underestimation and overestimation costs and was then incorporated in the wind-based economic emission dispatch problem.

The recent trend is directed towards the integration of ESSs into wind-thermal systems regarding the power dispatch problem. Recent studies [17, 18] have demonstrated that ESSs can decrease operational cost, improve the power system's reliability, and mitigate the impact of wind power fluctuations. Battery energy storage sizing technique has been intensively discussed in [19]. The study explored the relationship between the size of centralized battery storage and operation cost of distributed generation in a microgrid using economic power dispatch. It explained how optimal capacity storage within a specified investment cost and predefined system constraints could provide a significant reduction in operation cost. The battery storage of the study has also created opportunities for the microgrid to trade energy in the electricity market during off-peak times, which increases the benefits of the dispatchable generating units. A technique based on back-propagation neural network and multi-objective PSO was proposed in [17] for optimum sitting and sizing of ESSs in hybrid wind-diesel power networks. In [18], an ESS was utilized in the DEED problem when intermittency and uncertainty of the wind power is described by a chance constraint. Authors in [20] used Markov chain to simulate PV and wind power and then applied the PSO algorithm to dispatch the thermal units and ESSs' outputs. The DEED problem aims to find the optimum schedule of power generation in order to minimize simultaneously total production cost and emission of harmful gases. The focus of this paper is to solve the DEED problem incorporating wind farms and ESSs. The main contributions of this study are: 
- The problem is represented as a multi-objective problem and the SPEA is used to provide the best solutions. Total fuel cost and total emission functions are minimized simultaneously.

- To address the uncertainty of the wind power output, the energy balance is converted into a stochastic constraint.

- The effectiveness of the proposed method is verified on a ten-unit system and through comparison with other published optimization techniques. The simulation results show that the incorporation of wind power and ESSs into the DEED problem may decrease cost and emissions.

\section{MATHEMATICAL MODELING OF THE PROBLEM}

\section{A. Modeling of the Wind Power for the Dispatch Problem}

The uncertainty of the wind power is modeled by a chance constraint and a Probability Distribution Function (PDF) is used to describe the stochastic nature of the wind speed. The Weibull PDF given in (1) is proposed to describe the wind speed distribution. The corresponding Cumulative Distribution Function (CDF) is given in (2).

$$
\begin{array}{r}
f_{V}(v)=\frac{k}{c}\left(\frac{v}{c}\right)^{k-1} \exp \left[-\left(\frac{v}{c}\right)^{k}\right] \\
F_{V}(v)=\int_{0}^{v} f_{V}(\tau) d \tau=1-\exp \left(-\left(\frac{v}{c}\right)^{k}\right), v \geq 0
\end{array}
$$

where, $v$ is the wind speed. Parameters $k$ and $c$ are the shape and scale factors. These parameters depend on the geographical location of the wind source. Several techniques such as least squares fit method and method of moments have been suggested to estimate the values of $k$ and $c$ [15].

The probability to have wind power equal to or less than $w$ is given in (3) [18], where, $v_{\text {in }}, v_{\text {out }}$ and $v_{r}$ are cut-in, cut-out, and rated wind speeds respectively.

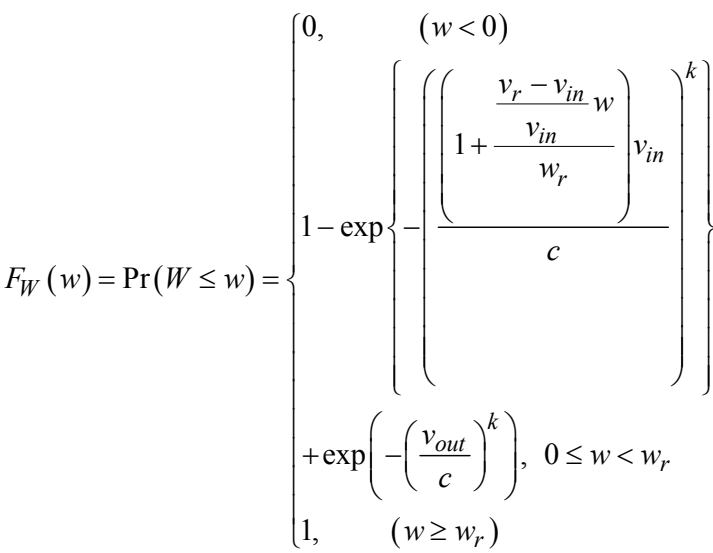

\section{B. Objective Functions and Constraints}

The DEED problem is formulated as an optimization problem, where the objective functions to be minimized are the total fuel cost FT and the total emission ET. These functions are [21]:

$$
\begin{gathered}
F_{T}=\sum_{h=1}^{H} \sum_{i=1}^{N} a_{i}+b_{i} P_{i, h}+c_{i}\left(P_{i, h}\right)^{2}+\left|d_{i} \sin \left\{e_{i}\left(\underline{P}_{i}-P_{i, h}\right)\right\}\right| \\
E_{T}=\sum_{h=1}^{H} \sum_{i=1}^{N} \alpha_{i}+\beta_{i} P_{i, h}+\gamma_{i}\left(P_{i, h}\right)^{2}+\eta_{i} \exp \left(\lambda_{i} P_{i, h}\right)
\end{gathered}
$$

where $a_{i}, b_{i}, c_{i}, d_{i}$, and $e_{i}$ are cost coefficients and $\alpha_{i}, \beta_{i}, \gamma_{i}, \eta_{i}$, and $\lambda_{i}$ are emission coefficients.

The previous objective functions are optimized under the following constraints:

1) Generation Limits

$$
\begin{array}{r}
\underline{P}_{i} \leq P_{i, h} \leq \bar{P}_{i}, i=1, \ldots, N \\
0 \leq W_{h} \leq w_{r}
\end{array}
$$

2) Ramp Rate Limits

$$
\begin{gathered}
P_{i, h-1}-P_{i, h} \leq U_{i}^{\text {down }} \\
P_{i, h}-P_{i, h-1} \leq U_{i}^{u p}
\end{gathered}
$$

where $U_{i}^{\text {down }}$ and $U_{i}^{u p}$ are the prohibited operating zones of unit $i$.

\section{3) Constraints Related to the ESS}

The Constraints of the ESS are described below. Equation (10) states the energy conversion. Equations (11), (12) and correspond to the limits of charging and discharging powers of the ESS and its storage capacity respectively.

$$
\begin{gathered}
E_{s, h}=E_{s, h-1}+\eta_{c} P_{s c, h} C H_{s, h} \Delta t-P_{s d, h} D C_{s, h} \Delta t / \eta_{s d} \\
0 \leq P_{s c, h} \leq k_{s c} \times E^{c a p} \\
0 \leq P_{s d, h} \leq k_{s d} \times E^{c a p} \\
0 \leq E_{s, h} \leq E^{c a p} \\
C H_{s, h} \times D C_{s, h}=0
\end{gathered}
$$

where $k_{s c} \in[0,1]$ and $k_{s d} \in[0,1]$.

\section{4) Energy Balance Constraint}

When wind power and ESSs are integrated into the DEED problem, the energy balance constraint can be described by a chance constraint in order to take into account the wind power intermittency. This constraint can be written as:

$$
F_{W}\left(P_{D, h}+\text { loss }_{h}-\sum_{i=1}^{N} P_{i, h}-W_{h}-P_{s c, h} C H_{s, h}+P_{s d, h} D C_{s, h}\right) \leq \zeta
$$

where $\operatorname{loss}_{h}$ is the total losses at hour $h$. It may be calculated using B-loss formula as given in (16): 


$$
\operatorname{loss}_{h}=\sum_{i=1}^{N} \sum_{j=1}^{N} P_{i, h} B_{i j} P_{j, h}+\sum_{i=1}^{N} B_{o i} P_{i, h}+B_{o o}
$$

where $B_{i j}, B_{o i}$ and $B_{o o}$ are the loss-coefficient matrices.

\section{PROPOSED ALGORITHM: SPEA APPROACH}

SPEA [21] is an elitist multi-objective evolutionary algorithm. A brief description of the SPEA is given in this section. For more detailed explanation, the interested reader can be referred to [21]. The main steps of an iteration of SPEA are:

- Step 1: Generate the initial population $P_{0}$ with $N_{\text {pop }}$ individuals, set the external archive $P_{0}^{\prime}=\varnothing$ and $t=0$.

- Step 2: Extract the Pareto solutions from $P_{t}$ referred as $\operatorname{PARETO}\left(P_{t}\right)$.

- Step 3: Set $P_{t}^{\prime}=P_{t}^{\prime} \cup \operatorname{PARETO}\left(P_{t}\right)$.

- Step 4: Set $P_{t}^{\prime}=\operatorname{PARETO}\left(P_{t}^{\prime}\right)$.

- Step 5: If $\operatorname{size}\left(P_{t}^{\prime}\right)>N^{\prime}$, reduce $P_{t}^{\prime}$ with the clustering method.

- Step 6: Assign the strength Pareto of each individual $i$ from $P_{t}^{\prime}$ defined as given in (17), where $n_{i}$ is the number of solutions from $P_{t}$ that are dominated by or are equal to the individual $i$.

$$
S_{i}=\frac{n_{i}}{N_{p o p}+1}
$$

- Step 7: Assign the fitness $f_{j}$ of each solution $X_{j}$ from the current population $P_{t}$ as given in the flowing equation:

$$
f_{j}=1+\sum_{i \prec j} S_{i}
$$

- Step 8: Combine the current population $P_{t}$ and the external archive $P_{t}^{\prime}$. Then, use selection, crossover, and mutation operations to generate the new population $P_{t+1}$.

- Step 9: Set $t=t+1$.

- Step 10: If $t<t_{\max }$ return to step 2. Else the archived members are the Pareto optimal solutions.

\section{SimUlation RESUltS}

\section{A. Presentation of the Ten-unit System}

In this section, the ten-unit system is used to prove the feasibility of the proposed optimization method for solving the DEED problem. All cited operating constraints are considered. The B-loss matrix of the ten-unit system is given in (19). Both cost and emissions are optimized individually and simultaneously according to the variation of the power demand $P_{D, h}$ in MW over a time period of one day, subdivided into 24 time intervals of one hour. Unit data are taken from [18]. Wind source and ESS parameters are as given in [18].

$$
B=10^{-4}\left[\begin{array}{llllllllll}
0.49 & 0.14 & 0.15 & 0.15 & 0.16 & 0.17 & 0.17 & 0.18 & 0.19 & 0.20 \\
0.14 & 0.45 & 0.16 & 0.16 & 0.17 & 0.15 & 0.15 & 0.16 & 0.18 & 0.18 \\
0.15 & 0.16 & 0.39 & 0.10 & 0.12 & 0.12 & 0.14 & 0.14 & 0.16 & 0.16 \\
0.15 & 0.16 & 0.10 & 0.40 & 0.14 & 0.10 & 0.11 & 0.12 & 0.14 & 0.15 \\
0.16 & 0.17 & 0.12 & 0.14 & 0.35 & 0.11 & 0.13 & 0.13 & 0.15 & 0.16 \\
0.17 & 0.15 & 0.12 & 0.10 & 0.11 & 0.36 & 0.12 & 0.12 & 0.14 & 0.15 \\
0.17 & 0.15 & 0.14 & 0.11 & 0.13 & 0.12 & 0.38 & 0.16 & 0.16 & 0.18 \\
0.18 & 0.16 & 0.14 & 0.12 & 0.13 & 0.12 & 0.16 & 0.40 & 0.15 & 0.16 \\
0.19 & 0.18 & 0.16 & 0.14 & 0.15 & 0.14 & 0.16 & 0.15 & 0.42 & 0.19 \\
0.20 & 0.18 & 0.16 & 0.15 & 0.16 & 0.15 & 0.18 & 0.16 & 0.19 & 0.44
\end{array}\right]
$$

\section{B. Implementation of the Proposed Method}

Two cases for the ten-unit system are considered in this sub-section. Case 1: only thermal units are considered and case 2: with WP and ESSs. Figure 1 shows the convergence of total cost and emissions for case 1, by using the proposed algorithm and the Non-dominated Sorting Genetic Algorithm (NSGA). Convergence is obtained after 100 iterations. Figure 2 shows the Pareto solutions of the static EED problem where the power demand equals to $1700 \mathrm{MW}$. From Figures 1 and 2 it is clear that the proposed method provides better solutions when compared to the NSGA.

(a)

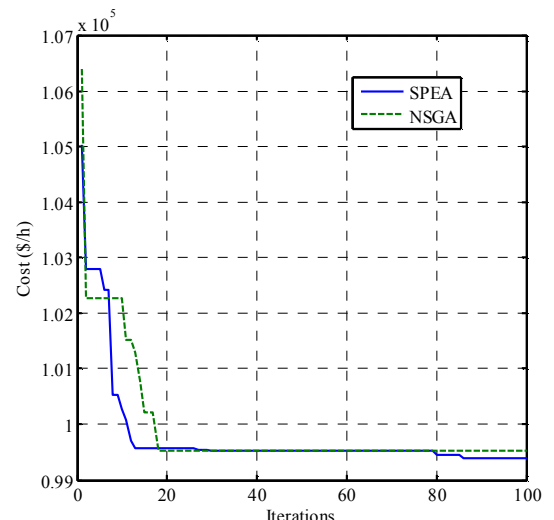

(b)

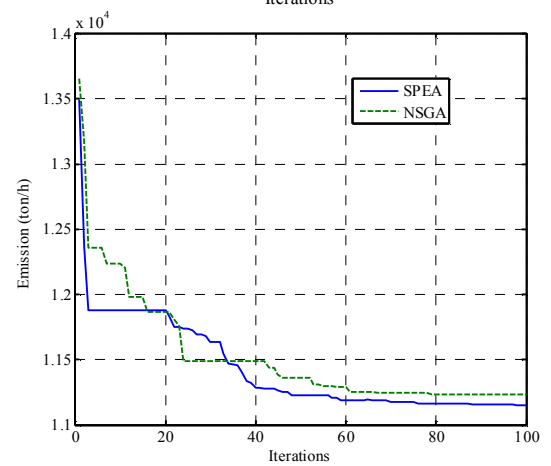

Fig. 1. Convergence of the objective functions: (a) cost, (b) emissions

Optimum compromise solutions under these two cases are shown in Tables II and III respectively. In this study, compromise solutions were obtained using fuzzy theory [23]. It is clear from these two Tables that all constraints were taken into account. In addition, the total production cost and the total emission are decreased when the wind energy source and the 
ESS are incorporated in the problem. The total production cost and the total emissions are 2530173.68\$ and 315022.47ton respectively for the first case and 2357551.22\$ and 266882.90ton respectively for the second case. The effect of the use of wind source and ESS is shown in Figure 3. It is clear that minimum production cost and minimum emissions are clearly reduced when wind power and ESSs are used.

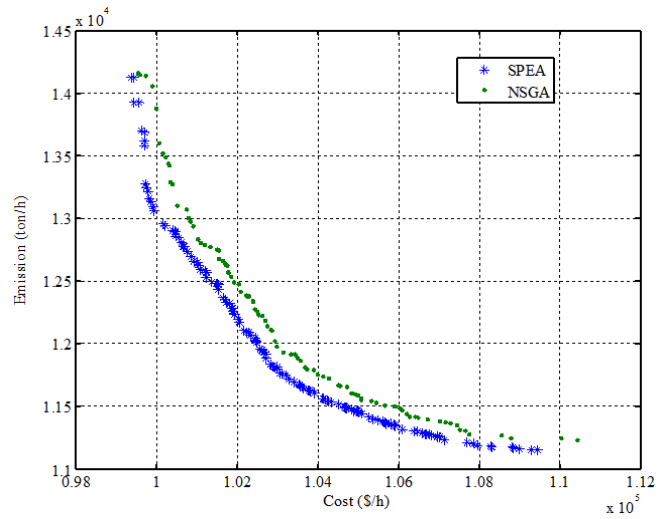

Fig. 2. Pareto solutions for $P_{D}=1700 \mathrm{MW}$

TABLE I. COMPARISON WITH OTHER TECHNIQUES FOR CASE 1

\begin{tabular}{|c|c|c|}
\hline Method & Minimum Cost & Minimum emissions \\
\hline SPEA & 2474311.14 & 295120.82 \\
\hline NSGAII & $2.5168 \times 106$ & $3.1740 \times 105$ \\
\hline IBFA & 2481733.3 & 295833.0 \\
\hline
\end{tabular}

It is clear from Table I that SPEA outperforms NSGAII [24] and IBFA [25] for both minimum cost and minimum emissions. (a)

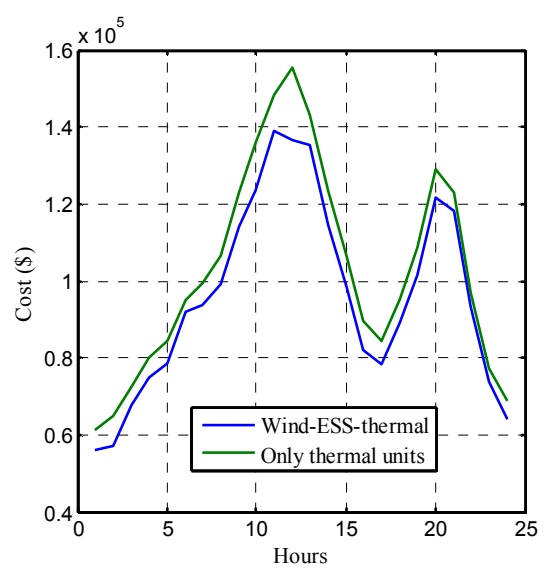

(b)

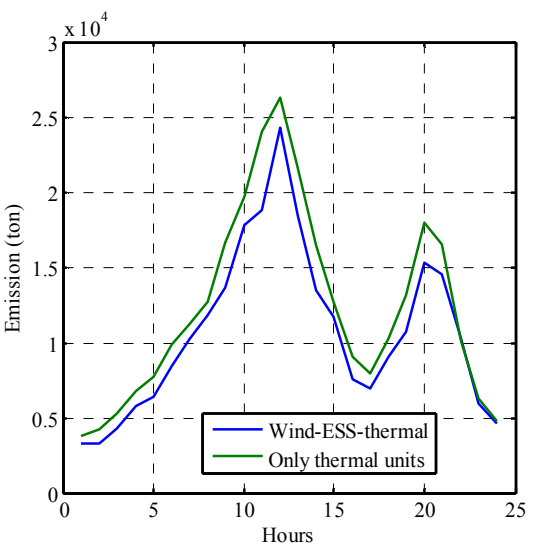

Fig. 3. Variation of minimum cost and emission over a single day

TABLE II. BEST COMPROMISE SOLUTION FOR CASE 1

\begin{tabular}{|c|c|c|c|c|c|c|c|c|c|c|c|}
\hline Hour & $\boldsymbol{P}_{D, h}$ & $P_{1}$ & $P_{2}$ & $P_{3}$ & $P_{4}$ & $P_{5}$ & $P_{6}$ & $\boldsymbol{P}_{7}$ & $P_{8}$ & $P_{9}$ & $P_{10}$ \\
\hline 1 & 1036 & 151.87 & 135.00 & 93.95 & 68.72 & 126.84 & 132.97 & 102.84 & 120.00 & 80.00 & 43.58 \\
\hline 2 & 1110 & 150.81 & 135.00 & 80.55 & 118.72 & 170.82 & 144.07 & 116.73 & 90.00 & 71.01 & 54.74 \\
\hline 3 & 1258 & 154.10 & 135.00 & 141.16 & 138.64 & 181.96 & 151.59 & 130.00 & 120.00 & 79.10 & 55.00 \\
\hline 4 & 1406 & 159.01 & 215.00 & 174.99 & 122.99 & 231.96 & 160.00 & 130.00 & 113.47 & 80.00 & 55.00 \\
\hline 5 & 1480 & 171.29 & 221.75 & 188.90 & 172.99 & 231.97 & 157.95 & 128.43 & 115.54 & 78.32 & 53.17 \\
\hline 6 & 1628 & 217.44 & 223.49 & 262.22 & 192.82 & 243.00 & 160.00 & 123.34 & 120.00 & 80.00 & 55.00 \\
\hline 7 & 1702 & 249.44 & 244.87 & 271.04 & 226.46 & 225.95 & 160.00 & 130.00 & 120.00 & 80.00 & 48.65 \\
\hline 8 & 1776 & 229.28 & 265.74 & 281.88 & 276.46 & 243.00 & 159.17 & 128.73 & 118.25 & 79.37 & 53.30 \\
\hline 9 & 1924 & 248.81 & 345.74 & 312.37 & 300.00 & 243.00 & 160.00 & 130.00 & 120.00 & 80.00 & 55.00 \\
\hline 10 & 2022 & 303.44 & 404.20 & 340.00 & 300.00 & 237.47 & 153.90 & 130.00 & 116.79 & 75.22 & 41.20 \\
\hline 11 & 2106 & 371.77 & 415.27 & 340.00 & 300.00 & 243.00 & 139.45 & 130.00 & 120.00 & 80.00 & 55.00 \\
\hline 12 & 2150 & 344.53 & 470.00 & 340.00 & 300.00 & 243.00 & 160.00 & 130.00 & 120.00 & 80.00 & 55.00 \\
\hline 13 & 2072 & 368.93 & 390.00 & 340.00 & 300.00 & 243.00 & 160.00 & 130.00 & 120.00 & 80.00 & 25.00 \\
\hline 14 & 1924 & 298.98 & 312.96 & 299.65 & 300.00 & 242.05 & 159.68 & 128.90 & 117.93 & 80.00 & 55.00 \\
\hline 15 & 1776 & 224.60 & 241.71 & 295.30 & 300.00 & 243.00 & 159.28 & 130.00 & 109.59 & 78.67 & 52.78 \\
\hline 16 & 1554 & 161.81 & 161.71 & 265.52 & 250.00 & 238.72 & 160.00 & 130.00 & 120.00 & 55.00 & 55.00 \\
\hline 17 & 1480 & 161.2867 & 165.25 & 190.72 & 242.21 & 242.21 & 160.00 & 128.15 & 120.00 & 55.00 & 54.83 \\
\hline 18 & 1628 & 198.24 & 155.05 & 270.72 & 292.21 & 243.00 & 160.00 & 130.00 & 120.00 & 55.00 & 52.24 \\
\hline 19 & 1776 & 272.35 & 235.05 & 283.41 & 299.61 & 235.61 & 160.00 & 130.00 & 116.50 & 53.27 & 49.63 \\
\hline 20 & 1972 & 346.63 & 315.05 & 307.59 & 300.00 & 243.00 & 160.00 & 130.00 & 120.00 & 70.28 & 55.00 \\
\hline 21 & 1924 & 305.13 & 308.83 & 327.88 & 300.00 & 230.08 & 159.72 & 109.96 & 119.88 & 78.89 & 55.00 \\
\hline 22 & 1628 & 227.10 & 228.83 & 247.88 & 250.00 & 243.00 & 160.00 & 96.77 & 120.00 & 48.89 & 55.00 \\
\hline 23 & 1332 & 153.70 & 148.83 & 171.15 & 200.00 & 193.00 & 119.83 & 126.77 & 120.00 & 78.89 & 51.95 \\
\hline 24 & 1184 & 154.35 & 135.00 & 106.67 & 118.72 & 163.66 & 152.62 & 130.00 & 119.97 & 75.46 & 52.98 \\
\hline Total cost $(\$)$ & \multicolumn{11}{|c|}{2530173.68} \\
\hline Total emission (ton) & \multicolumn{11}{|c|}{$\mathbf{3 1 5 0 2 2 . 4 7}$} \\
\hline Total losses (MW) & \multicolumn{11}{|c|}{1302.82} \\
\hline
\end{tabular}


TABLE III. BEST COMPROMISE SOLUTION FOR CASE 2

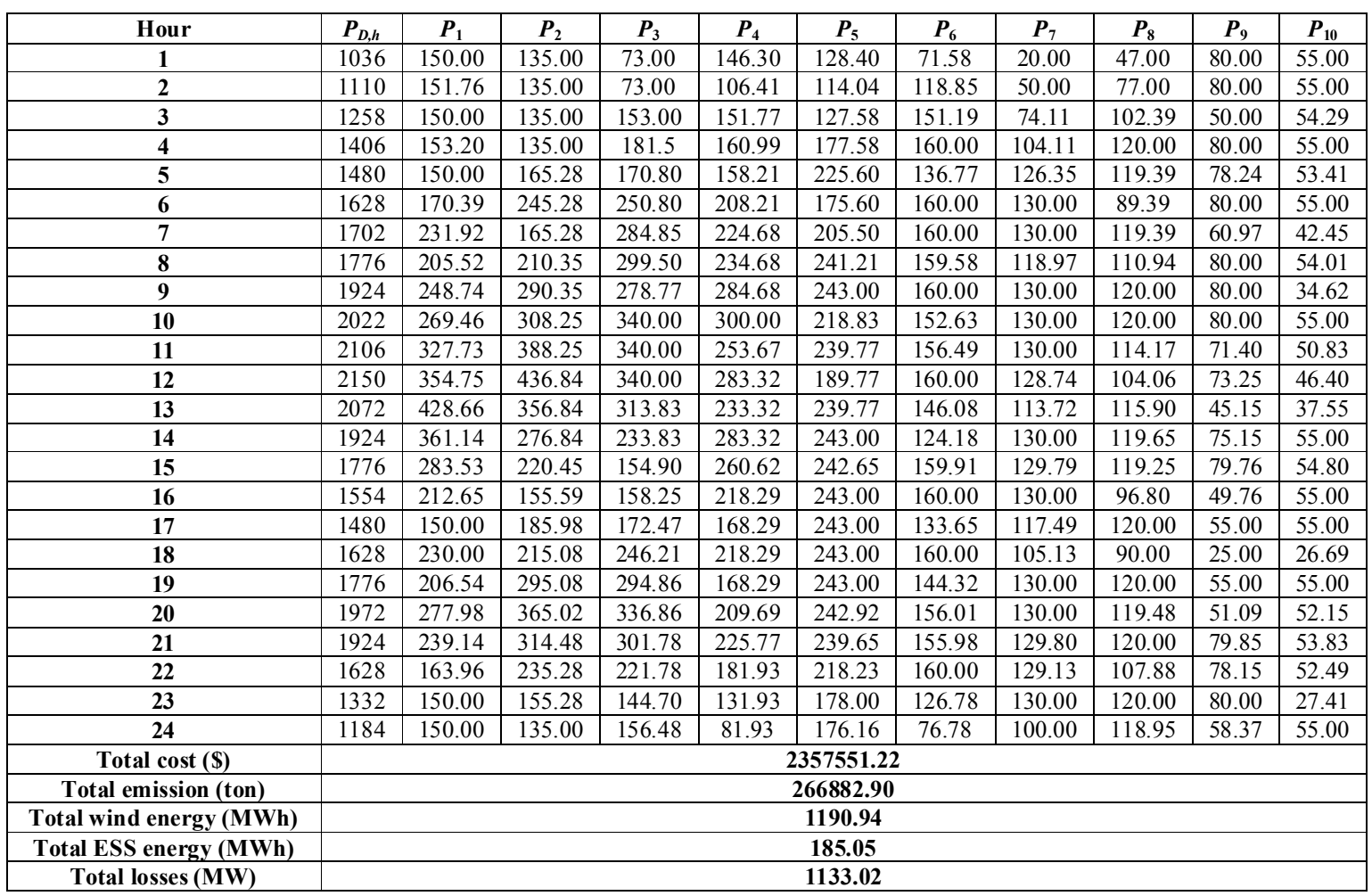

\section{CONCLUSION}

During the last decades, the high penetration of wind energy has created difficulties in energy dispatching due to the stochastic availability of wind power. Thus it is required to handle these random characteristics of energy sources. In this study, a method based on the SPEA technique is proposed for solving the DEED problem incorporating wind power. In order to avoid the overestimation penalty and underestimation costs, the wind power output is described by a chance constraint. Moreover, ESSs are added to the power network to increase the penetration of the wind energy. The effectiveness of the proposed method is verified on a ten-unit system. Simulation results show that the incorporation of ESS into the DEED problem can decrease clearly cost and emissions.

\section{ACKNOWLEDGMENT}

The author would like to thank the Deanship of the Scientific Research of University of Hail, Saudi Arabia for funding and supporting this research under the project (BA1917).

\section{NOMENCLATURE}

$\begin{array}{cl}f_{V}(\bullet) & \text { Probability density function (PDF) } \\ F_{V}(\bullet) & \text { Cumulative distribution function }(\mathrm{CDF}) \\ v & \text { Wind speed in } \mathrm{m} / \mathrm{s} \\ \zeta & \text { Threshold tolerance } \\ F_{T} & \text { Total fuel cost in } \$ . \\ E_{T} & \text { Total emission in tons } \\ P_{i, h} & \text { Output power of unit } i \text { at hour } h\end{array}$

$$
\begin{array}{cl}
P_{i}, \bar{P}_{i} & \text { Lower and upper generation limits of unit } i \\
N & \text { Number of thermal units } \\
W_{h} & \text { Wind power output at hour } h \text { in MW } \\
E_{s, h} & \text { Stored energy of the ESS at hour } h \\
P_{s c, h} & \text { Charging power at hour } h \text { in MW } \\
P_{s d, h} & \text { Discharging power at hour } h \text { in MW } \\
\eta_{c} \text { and } \eta_{s d} & \text { ESS charging and discharging efficiency, respectively } \\
C H_{s, h} \text { and } D C_{s, h} & \text { Charging and discharging status at hour } h \\
E^{c a p} & \text { ESS capacity in MWh } \\
l o s s_{h} & \text { Total losses at hour } h \\
P_{D, h} & \text { Demand power at hour } h
\end{array}
$$

\section{REFERENCES}

[1] L. Han, R. Zhang, K. Chen, "A coordinated dispatch method for energy storage power system considering wind power ramp event", Applied Soft Computing, Vol. 84, Article ID 105732, 2019

[2] W. Wang, R. Ma, H. Xu, H. Wang, K. Cao, L. Chen, Z. Ren, "Method of energy storage system sizing for wind power generation integration", IEEE PES Asia-Pacific Power and Energy Engineering Conference, Xi'an, China, October 25-28, 2016

[3] J. Wu, Y. Lin, "Economic dispatch including wind power injection", in: Proceedings of ISES World Congress 2007, Vol. I-V, Springer, 2007

[4] F. Benhamida, Y. Salhi, I. Ziane, S. Souag, R. Belhachem, A. Bendaoud, "A PSO algorithm for the economic load dispatch including a renewable wind energy", 3rd International Conference on Systems and Control, Algiers, Algeria, October 29-31, 2013

[5] K. K. Vishwakarma, H. M. Dubey, "Simulated annealing based optimization for solving large scale economic load dispatch problems", International Journal of Engineering Research \& Technology, Vol. 1, No. 3, pp. 1-8, 2012 
[6] R. V. Pandi, B. K. Panigrahi, "Dynamic economic load dispatch using hybrid swarm intelligence base harmony search algorithm", Expert Systems with Applications, Vol. 38, No. 7, pp. 8509-8514, 2011

[7] M. Younes, R. L. Kherfene, F. Khodja, "Environmental/economic power dispatch problem/renewable energy using firefly algorithm", International Conference on Environment, Energy, Ecosystems and Development, Venice, Italy, September 28-30, 2013

[8] P. K. Roy, S. Hazra, "Economic emission dispatch for wind-fossil-fuelbased power system using chemical reaction optimisation", International Transactions on Electrical Energy Systems, Vol. 25, No. 12, pp. 32483274, 2014

[9] Z. Wang, C. Shen, F. Liu, "A conditional model of wind power forecast errors and its application in scenario generation", Applied Energy, Vol. 212, pp. 771-785, 2018

[10] L. Han, R. Zhang, X. Wang, Y. Dong, "Multi-time scale rolling economic dispatch for wind/storage power system based on forecast error feature extraction", Energies, Vol. 11, No. 8, Article ID 2124, 2018

[11] Q. Wang, Y. Guan, J. Wang, "A chance-constrained two-stage stochastic program for unit commitment with uncertain wind power output", IEEE Transactions on Power Systems, Vol. 27, No. 1, pp. 206-215, 2012

[12] M. A. O. Vazquez, D. S. Kirschen, "Estimating the spinning reserve requirements in systems with significant wind power generation penetration", IEEE Transactions on Power Systems, Vol. 24, No. 1, pp. 114-124, 2009

[13] P. Xiong, P. Jirutitijaroen, C. Singh, "A distributionally robust optimization model for unit commitment considering uncertain wind power generation", IEEE Transactions on Power Systems, Vol. 32, No. 1, pp. 39-49, 2017

[14] Y. Hu, Y. Li, M. Xu, L. Zhou, M. Cui, "A chance-constrained economic dispatch model in wind-thermal-energy storage system", Energies, Vol. 10, No. 3, Article ID 326, 2017

[15] X. Liu, W. Xu, "Economic load dispatch constrained by wind power availability: A here-and-now approach", IEEE Transactions on Sustainable Energy, Vol. 1, No. 1, pp. 2-9, 2010

[16] S. Hazra, P. K. Roy, "Quasi-oppositional chemical reaction optimization for combined economic emission dispatch in power system considering wind power uncertainties", Renewable Energy Focus, Vol. 31, pp. 4562,2019

[17] H. Lan, H. Yin, S. Wen, Y. Y. Hong, D. C. Yu, L. Zhang, "Electrical energy forecasting and optimal allocation of ESS in a hybrid wind-diesel power system", Applied Sciences, Vol. 7, No. 2, Article ID 155, 2017

[18] M. H. Alham, M. Elshahed, D. K. Ibrahim, E. E. D. A. E. Zahab, “A dynamic economic emission dispatch considering wind power uncertainty incorporating energy storage system and demand side management", Renewable Energy, Vol. 96, pp. 800-811, 2016

[19] K. Alqunun, P. A. Crossley, "Rated energy impact of BESS on total operation cost in a microgrid", International Conference on Smart Energy Grid Engineering, Oshawa, Canada, August 21-24, 2016

[20] B. Xiao, Y. Zhang, J. Han, D. Liu, M. Wang, G. Yan, “A multi-energy complementary coordinated dispatch method for integrated system of wind-photovoltaic-hydro-thermal-energy storage", International Transactions on Electrical Energy Systems, Vol. 29, No. 7, Article ID e12005, 2019

[21] A. Torchani, A. Boudjemline, H. Gasmi, Y. Bouazzi, T. Guesmi, "Dynamic economic/environmental dispatch problem considering prohibited operating zones", Engineering, Technology \& Applied Science Research, Vol. 9, No. 5, pp. 4586-4590, 2019

[22] E. Zitzler, L. Thiele, "Multiobjective evolutionary algorithms: A comparative case study and the strength pareto approach", IEEE Transactions on Evolutionary Computation, Vol. 3, No. 4, pp. 257-271, 1999

[23] I. Marouani, A. Boudjemline, T. Guesmi, H. H. Abdallah, "A modified artificial bee colony for the nonsmooth dynamic economic/environmental dispatch", Engineering, Technology \& Applied Science Research, Vol. 8, No. 5, pp. 3321-3328, 2018

[24] M. Basu, "Dynamic economic emission dispatch using nondominated sorting genetic algorithm-II", International Journal of Electrical Power \& Energy Systems, Vol. 30, No. 2, pp. 140-149, 2008
[25] N. Pandit, A. Tripathi, S. Tapaswi, M. Pandit, "An improved bacterial foraging algorithm for combined static/dynamic environmental economic dispatch", Applied Soft Computing, Vol. 12, No. 11, pp. $3500-3513,2012$ 